\title{
ANLN Directly Interacts with RhoA to Promote Doxorubicin Resistance in Breast Cancer Cells
}

This article was published in the following Dove Press journal:

Cancer Management and Research

\section{Feng Wang* \\ Zhen Xiang* \\ Teng Huang* \\ Min Zhang \\ Wei-Bing Zhou}

Department of Oncology, Xiangya Hospital, Central South University, Changsha, Hunan 410008, People's Republic of China

*These authors contributed equally to this work
Correspondence: Wei-Bing Zhou Department of Oncology, Xiangya Hospital, Central South University, No. 87 Xiangya Road, Changsha, Hunan 410008, People's Republic of China

Tel +86-73l-89753733

Email zhouweibing298@csu.edu.cn
Background: Chemotherapy resistance is the leading cause of cancer treatment failure. This research was conducted to explore a potential link between actin-binding protein anillin (ANLN) and doxorubicin resistance in breast cancer.

Materials and Methods: We compared ANLN expression and 50\% inhibition concentration (IC50) of doxorubicin in human breast cancer cells (MDA-MB-231) and human breast cancer cells with doxorubicin resistance (MDA-MB-231/ADM). Co-immunoprecipitation was used to investigate the interaction between ANLN and RhoA. The cell viability, apoptosis, gene and protein expression were estimated by MTT, flow cytometry, quantitative real-time PCR and western blot.

Results: The doxorubicin resistance in MDA-MB-231/ADM cells (IC50 = $19.40 \pm 1.16 \mu \mathrm{g}$ ) $\mathrm{mL}$ ) was significantly higher than that in MDA-MB-231 cells (IC50 $=1.65 \pm 0.23 \mu \mathrm{g} / \mathrm{mL}$ ). ANLN was up-regulated in MDA-MB-231/ADM cells compared to MDA-MB-231 cells. Furthermore, ANLN overexpression promoted cell viability and inhibited apoptosis of MDA-MB-231 cells. The gene and protein expression of multidrug resistance (MDR1) and cancer resistance protein (BCRP) were enhanced by ANLN overexpression in MDA-MB -231 cells. ANLN silencing suppressed cell viability and the expression of MDR1 and BCRP and facilitated apoptosis in MDA-MB-231/ADM cells. Moreover, ANLN promoted RhoA activation by interacting with RhoA. ANLN up-regulation enhanced cell viability and the expression of MDR1 and BCRP and decreased apoptosis of MDA-MB-231 cells. The influence conferred by ANLN overexpression was effectively abolished by $\mathrm{C} 3$ transferase. Conclusion: This work revealed that ANLN promoted doxorubicin resistance in breast cancer cells by activating RhoA. Thus, our study suggests a novel target for breast cancer treatment.

Keywords: ANLN, RhoA, doxorubicin resistance, breast cancer, RhoA, GTPase, patient stratification

\section{Introduction}

Breast cancer is one of the most common malignant tumors in women, which accounting for $30 \%$ of female malignancies. Breast cancer is also the second leading cause of death for women in developed countries. ${ }^{1}$ In the past decade, chemotherapy, endocrine therapy and targeted therapy have developed rapidly in breast cancer treatment. ${ }^{2,3}$ In particular, chemotherapy has become the first choice for breast cancer adjuvant therapy and rescue treatment. ${ }^{4}$ Despite breast cancer treatment has made extraordinary progress, chemotherapy has various problems in application. ${ }^{5}$ The main factor limiting the effectiveness of chemotherapy is the resistance of cancer cells to chemotherapy drugs. ${ }^{6}$ Anthracyclines are widely used 
in breast cancer chemotherapy, which improve survival rate and reduce recurrence rate and mortality in adjuvant chemotherapy after breast cancer surgery. However, more and more patients are developing anthracycline resistance, which is closely associated with treatment failure. Therefore, there is an urgent need to address the problems caused by anthracycline resistance in breast cancer.

Ras homologous family A (RhoA) is a small molecule $\mathrm{G}$ protein/GTPase that circulates between inactive guanylate (GDP) and active guanylate (GTP) forms. ${ }^{7}$ Previous studies have provided important information on the role of RhoA in tumor chemotherapy resistance. RhoA regulates the cytoskeleton by activating effector proteins such as ROCK, thereby participating in invasion and metastasis of tumor cells. ${ }^{8}$ In addition, RhoA plays a crucial role in regulating tumor drug resistance. ${ }^{9}$ Surveys such as that conducted by Yoon et al have shown that RhoA is highly expressed in diffuse-type gastric adenocarcinoma, and RhoA knockdown promotes the sensitivity of diffuse gastric adenocarcinoma spheroid cells to cisplatin chemotherapy. ${ }^{10}$ RhoA is up-regulated in colorectal cancer cells with irinotecan resistance. RhoA silencing enhances the sensitivity of colorectal cancer cells to irinotecan by inhibiting the expression of resistant genes such as multidrug resistance (MDR1), MRP1, and glutathione S-transferase pi 1 (GSTP1). ${ }^{11}$ MiR-31 suppresses cell proliferation, migration and invasion of gastric cancer cells and facilitates the sensitivity of gastric cancer cells to 5-fluorouracil by inhibiting RhoA expression. ${ }^{12}$

Actin-binding protein anillin (ANLN) is mainly involved in mitosis. Existing research recognizes that ANLN is associated with the occurrence and development of cancer. ANLN is highly expressed in breast cancer tissues and cells, and ANLN can be used as a biomarker for early diagnosis of breast cancer. ${ }^{13}$ ANLN silencing inhibits proliferation and migration of breast cancer. ${ }^{14}$ ANLN is also closely associated with anthracycline resistance of breast cancer. Breast cancer patients with high expression of ANLN exhibit poor prognosis and anthracycline resistance. ${ }^{15}$ Our previous research also reveals that knockdown of ANLN leads to a decrease in cell viability and migration of breast cancer. Recent evidence suggests that ANLN directly interacts with RhoA and promote the activation of RhoA in lung cancer. ${ }^{16}$ Thus, we chose two human breast cancer cell lines, MDA-MB-231 and MDAMB-231/ADM, to explore the mechanism of action of ANLN in the chemoresistance of breast cancer cells to doxorubicin. MDA-MB-231 cells are derived from pleural effusion of a female patient with metastatic breast cancer. ${ }^{17}$ MDA-MB-231/ADM cells are human breast cancer cells with doxorubicin resistance. ${ }^{18}$ We speculate that in breast cancer, ANLN promotes the activation of RhoA by interacting with RhoA, thereby regulating the doxorubicin resistance of breast cancer.

\section{Materials and Methods Cell Culture}

Human breast cancer cells (MDA-MB-231) and human breast cancer cells with doxorubicin resistance (MDAMB-231/ADM) were obtained from public cell banks (ATCC, Manassas, VA, USA). These cells were cultured in Dulbecco's Modified Eagle Medium (DMEM) (Sangon Biotech, Shanghai, China) high glucose supplemented with $10 \%$ fetal bovine serum (Sangon Biotech) and 1\% penicillin/streptomycin. All the cells were incubated in a humidified atmosphere at $37^{\circ} \mathrm{C}$ and $5 \% \mathrm{CO}_{2}$.

\section{Drug Treatment}

To examine the $50 \%$ inhibition concentration (IC50) of doxorubicin to MDA-MB-231 and MDA-MB-231/ADM cells, these cells were treated with different concentrations of doxorubicin at $37^{\circ} \mathrm{C}$ and $5 \% \mathrm{CO}_{2}$ for $24 \mathrm{~h}$. Then, MDAMB-231 and MDA-MB-231/ADM cells were treated with $5 \mu \mathrm{g} / \mathrm{mL}$ doxorubicin. MDA-MB-231 and MDA-MB-231/ ADM cells were treated with $\mathrm{C} 3$ transferase $(1 \mathrm{~g} / \mathrm{mL})$ at $37^{\circ} \mathrm{C}$ for $4 \mathrm{~h}$ to inhibit RhoA activity.

\section{Cell Transfection}

Plasmid vector pcDNA3.1-ANLN was constructed by RiboBio (Guangzhou, China) via standard molecular cloning approaches. The empty vector (pcDNA3.1-NC) served as control (Ctrl). Small interfering RNAs specific targeting ANLN (si-ANLN) and its negative control (NC) were purchased from RiboBio. Plasmids were transfected into the cells MDA-MB-231 or MDA-MB-231/ADM cells using Lipofectamine 2000 Transfection Reagent (Invitrogen, Carlsbad, CA, USA) according to the instruction of the test kits.

\section{MTT Assay}

The MTT assay was used to estimate cell viability the IC50 of doxorubicin to MDA-MB-231 and MDA-MB $-231 / \mathrm{ADM}$ cells. The cells at $\log$ phase were seeded into 96-well plates $\left(5 \times 10^{3} /\right.$ well $)$ and treated with $5 \mu \mathrm{g} / \mathrm{mL}$ doxorubicin at $37^{\circ} \mathrm{C}$ and $5 \% \mathrm{CO}_{2}$ for $24 \mathrm{~h}$. After that, MTT 
reagent $(20 \mu \mathrm{L})$ was added into each well, and the cells cultured for $4 \mathrm{~h}$ in an incubator at $37^{\circ} \mathrm{C}$. Then, supernatant was discarded and dimethyl sulfoxide (DMSO) reagent $(100 \mu \mathrm{L})$ was added into each well. The absorbance of samples was detected at $590 \mathrm{~nm}$ wavelength using enzyme-labeled instrument (Thermo Fisher Scientific, Waltham, MA, USA). The IC50 of doxorubicin to MDAMB-231 and MDA-MB-231/ADM cells were calculated.

\section{Flow Cytometry}

The apoptosis of the cells after treated with doxorubicin was measured by flow cytometry. The cells were collected by centrifuging for 5 minutes at the speed of $500 \times \mathrm{g}, 4^{\circ} \mathrm{C}$. The cells were washed with pre-cooling PBS for 2 times after treated with doxorubicin for $24 \mathrm{~h}$. The cells were then resuspended in the Annexin $\mathrm{V}$ Binding buffer. The cell suspension was dyed with Annexin V-FITC and PI and plunged into darkness at room temperature for $15 \mathrm{~min}$. Then, the cell suspension was mixed with Annexin $\mathrm{V}$ Binding buffer and put on ice. The apoptosis rate of cells was determined by flow cytometry in an hour. The assay was performed according to the instruction of Annexin V-FITC/PI Cell Apoptosis Detection Kit (TransGen Biotech, Beijing, China).

\section{Quantitative Real-Time PCR (qRT-PCR)}

QRT-PCR was used to measure the expression intensity of different genes. Total RNA was extracted from cells using RNAprep Pure Tissue Kit (Tiangen Biotech, Beijing, China). The purity of RNA was detected using NanoDrop 2000 spectrophotometer (Thermo Fisher Scientific). The quantity of RNA was determined by agarose gel electrophoresis. The RNA was reversely transcribed to complementary DNA using PrimeScript ${ }^{\mathrm{TM}}$ RT Reagent Kit (Takara, Tokyo, Japan). QRT-PCR was carried out using SYBR Green PCR Mix Kit (Takara) according to the instruction. The results were analyzed using the $\Delta \Delta \mathrm{CT}$ (cycle threshold) method for quantification.

\section{Co-Immunoprecipitation (Co-IP)}

Co-IP assay was used to detect the interaction between ANLN and RhoA in MDA-MB-231 and MDA-MB-231/ ADM cells. MDA-MB-231 and MDA-MB-231/ADM cells were co-transfected with pCMV-cMyc-ANLN and pCMVGFP-RhoA. The modified cells were obtained by centrifugation after washed with precooling phosphate buffer saline (PBS) for 2 times. The cells were treated with lysate and to serve it on ice for $30 \mathrm{~min}$ to lyse cells. Then, the cell supernatant was obtained by centrifugation. The cell supernatant of samples was incubated with antibody RhoA at $4{ }^{\circ} \mathrm{C}$ for $4 \mathrm{~h}$ after mixed with precooling PBS. (Input group: cell lysate; Output group: the cell supernatant after immunoprecipitation.) The antigen-antibody complex was incubated with $40 \mu \mathrm{L}$ protein $\mathrm{A} / \mathrm{G}$-beads at $4^{\circ} \mathrm{C}$ for $4 \mathrm{~h}$. The antigenantibody-beads complexes were obtained by centrifugation after washed with elution buffer. The levels of RhoA-GTP and total RhoA were determined by Western blot (WB). The assay was performed according to the instruction of RhoA Activation Assay Kit (NewEast Biosciences, Wuhan, China).

\section{WB}

Total protein was extracted from cells using Tissue or Cell Total Protein Extraction Kit (Sangon Biotech). Equivalent protein from different samples was separated by protein electrophoresis, following by transformation onto polyvinylidene fluoride (PVDF) membranes (Merck Millipore, Billerica, MA, USA). The membranes were incubated with anti-rabbit GFP, cMyc, MDR1 or BCRP (cancer resistance protein) (1:1000, Abcam, Cambridge, UK) at $4^{\circ} \mathrm{C}$ overnight after immersed into sealed liquid. After the membranes were washed with tris buffered saline containing $0.5 \%(\mathrm{v} / \mathrm{v})$ Tween 20 (TBST) for several times, goat antimouse IgG antibody (1:1000, Abcam) labeled with horseradish peroxidase were incubated with the membranes as a secondary antibody. Anti-mouse $\beta$-actin antibody (1:1000, Abcam) was used as a reference protein for normalization. The gray levels of the protein bands were examined by Image $\mathrm{J}$ software.

\section{Statistical Analysis}

All experiments were independently repeated at least 3 times. All values were exhibited as mean \pm standard deviation and analyzed by SPSS 22.0 statistical software (SPSS, USA). For comparison of two groups, a two-tailed Student's $t$ test was used. Comparison of multiple groups was made using a one- or two-way ANOVA. $P<0.05$ was considered statistically significant.

\section{Results}

\section{ANLN Overexpression Promotes Doxorubicin Resistance in MDA-MB-23I Cells}

In order to investigate the IC50 of doxorubicin to MDA-MB -231 and MDA-MB-231/ADM cells, we performed MTT assay. The IC50 of doxorubicin to MDA-MB-231 and 
MDA-MB-231/ADM cells was $1.65 \pm 0.23 \mu \mathrm{g} / \mathrm{mL}$ and $19.40 \pm 1.16 \mu \mathrm{g} / \mathrm{mL}$, suggesting that the doxorubicin resistance in MDA-MB-231/ADM cells was significantly higher than that in MDA-MB-231 cells (Table 1). Subsequently, to examine the involvement of ANLN in breast cancer, we analyzed its expression in MDA-MB-231 and MDA-MB -231/ADM cells. Compared with MDA-MB-231 cells, ANLN expression was characterized by a significantly higher in MDA-MB-231/ADM cells (Figure 1A). Then, MDA-MB-231 cells were transfected with pcDNA3.1-ANLN to induce ANLN up-regulation. Figure $1 B$ shows that ANLN is significantly up-regulated in MDAMB-231 cells after transfected with pcDNA3.1- ANLN. Next, we validated the influence of ANLN overexpression on cell viability and apoptosis by MTT and flow cytometry assay. The cell viability was severely suppressed in MDAMB-231 cells after doxorubicin treatment. ANLN overexpression caused a boost in cell viability of doxorubicintreated MDA-MB-231 cells (Figure 1C). Instead, doxorubicin treatment led to an increase in apoptosis of MDA-MB -231 cells, which was effectively abolished by ANLN upregulation (Figure 1D). Furthermore, the expression of drug resistance-related genes, MDR1 and BCRP, in MDA-MB -231 cells was assessed by qRT-PCR and WB. The gene and protein expression of MDR1 and BCRP was severely downregulated in MDA-MB-231 cells after doxorubicin treatment. And ANLN overexpression caused an obvious increase in the gene and protein expression of MDR1 and BCRP in doxorubicin-treated MDA-MB-231 cells (Figure $1 \mathrm{E}$ and $\mathrm{F}$ ). Thus, these results indicated that ANLN overexpression promoted doxorubicin resistance in MDA-MB -231 cells.

\section{ANLN Silencing Inhibits Doxorubicin Resistance in MDA-MB-23I/ADM Cells}

To further investigate the role of ANLN in breast cancer, MDA-MB-231/ADM cells were transfected with si-ANLN to induce ANLN knockdown in MDA-MB-231/ADM cells. ANLN silencing led to a decrease of ANLN

Table I The IC50 of Doxorubicin to MDA-MB-23I and MDAMB-23I/ADM Cells

\begin{tabular}{|l|l|}
\hline Group & IC50 $(\mu \mathrm{g} / \mathrm{mL})$ \\
\hline MDA-MB-23I & $1.65 \pm 0.23$ \\
MDA-MB-23I/ADM & $19.40 \pm 1.16^{* *}$ \\
\hline
\end{tabular}

Note: ${ }^{*} P<<0.01$ compared with the MDA-MB-23I group. expression in the MDA-MB-231/ADM cells (Figure 2A). Then, the cell viability of MDA-MB-231/ADM cells was analyzed by MTT assay, revealing that doxorubicin treatment had no effect on cell viability of MDA-MB-231/ ADM cells. ANLN-silenced MDA-MB-231/ADM cells showed the dramatic decrease in cell viability after doxorubicin treatment (Figure 2B). Besides, flow cytometry confirmed that doxorubicin treatment caused an increase in apoptosis of MDA-MB-231/ADM cells. ANLN knockdown notably enhanced apoptosis of MDA-MB-231/ADM cells after doxorubicin treatment (Figure 2C). Moreover, the gene and protein expression of MDR1 and BCRP was estimated by qRT-PCR and WB. Doxorubicin treatment resulted in a pronounced increase in the gene and protein expression of MDR1 and BCRP in MDA-MB-231/ADM cells. The gene and protein expression of MDR1 and BCRP were severely down-regulated by ANLN knockdown in MDA-MB-231/ADM cells after doxorubicin treatment (Figure 2D and E). These data taken together demystified that ANLN silencing inhibited doxorubicin resistance in MDA-MB-231/ADM cells.

\section{ANLN Promotes RhoA Activation by Interacting with RhoA}

To verify if ANLN directly interacted with RhoA in MDA-MB-231 and MDA-MB-231/ADM cells, we performed Co-IP assay. Compared with the Vector group, the levels of GTP-RhoA were notably increased in the ANLN group (Figure $3 \mathrm{~A}$ and $\mathrm{B}$ ). Therefore, these data verified that ANLN promoted RhoA activation by interacting with RhoA.

\section{ANLN Promotes Doxorubicin Resistance in MDA-MB-23 I Cells by Activating RhoA} We speculated that ANLN promoted doxorubicin resistance in breast cancer cells by activating RhoA. To verify this hypothesis, ANLN-upregulated MDA-MB-231 cells were treated with $\mathrm{C} 3$ transferase (RhoA inhibitor) and doxorubicin. MTT data showed that ANLN overexpression caused a boost in cell viability of MDA-MB-231 cells after doxorubicin treatment. The cell viability was severely decreased in MDA-MB-231 cells after doxorubicin combined with C3 transferase treatment. And the promoting effect of ANLN overexpression on cell viability in doxorubicin-treated MDA-MB-231 cells was dramatically repressed by $\mathrm{C} 3$ transferase treatment (Figure 4A). Then, the modified MDA-MB -231 cells were analyzed for apoptosis by flow cytometry, 

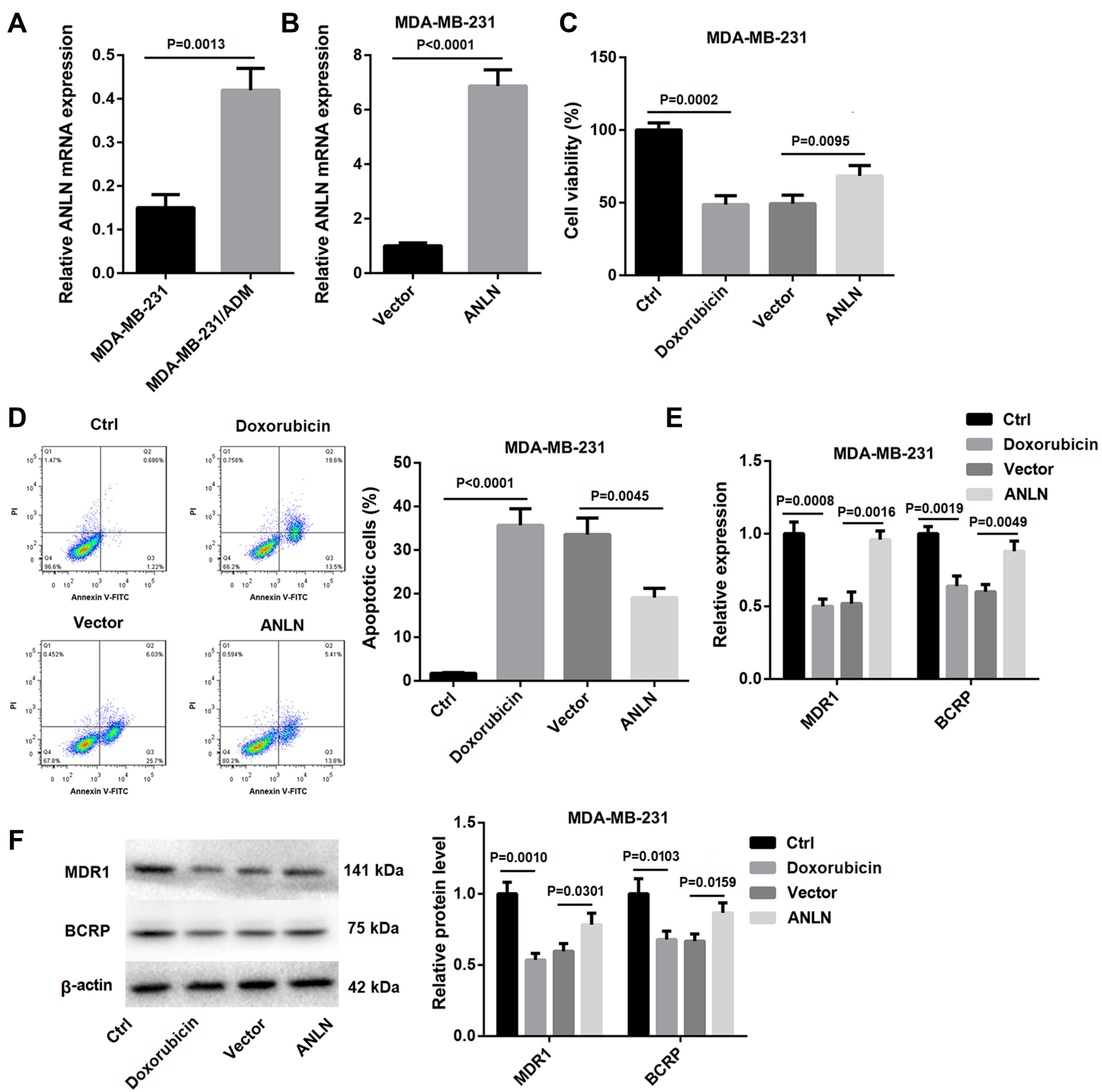

Figure I ANLN overexpression promotes doxorubicin resistance in MDA-MB-23। cells. (A) QRT-PCR was performed to estimate the expression of ANLN in MDA-MB $-23 \mathrm{I}$ and MDA-MB-23I/ADM cells. MDA-MB-23I cells were transected with pcDNA3.I-ANLN or pcDNA3.I-NC. (B) QRT-PCR was performed to assess the expression of ANLN in the modified MDA-MB-23। cells. The wild and modified MDA-MB-23I cells were treated with doxorubicin. Normal MDA-MB-23I cells served as control. (C) MTT assay was performed to explore cell viability of the MDA-MB-23I cells. (D) Flow cytometry was performed to estimate apoptosis of the MDA-MB-23I cells. (E) The expression of MDRI and BCRP in the MDA-MB-23I cells was assessed by qRT-PCR. (F) WB was performed to assess the expression of MDRI and BCRP in the MDA-MB -23 I cells.

revealing that ANLN overexpression led to a decrease in apoptosis of doxorubicin-treated MDA-MB-231 cells. However, C3 transferase treatment notably promoted apoptosis in MDA-MB-231 cells after doxorubicin treatment, which was abolished by ANLN overexpression (Figure 4B). Furthermore, the influence of ANLN overexpression on the gene and protein expression of MDR1 and BCRP was assessed by qRT-PCR and WB. ANLN overexpression resulted in a boost in the gene and protein expression of MDR1 and BCRP in MDA-MB-231 cells after doxorubicin treatment. C3 transferase treatment severely inhibited the gene and protein expression of MDR1 and BCRP in doxorubicin-treated MDA-MB-231 cells. And the influence conferred by ANLN overexpression was dramatically 


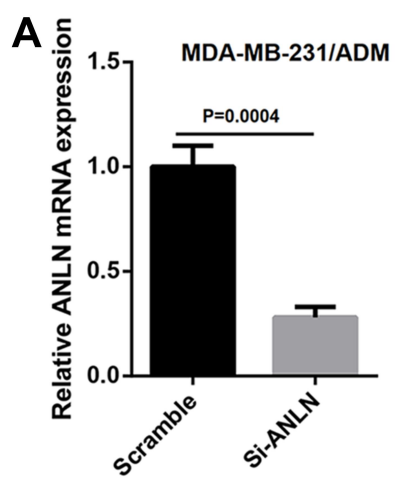

C

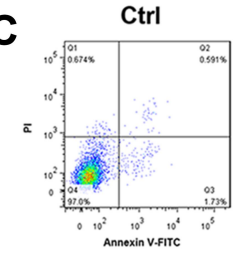

Doxorubicin
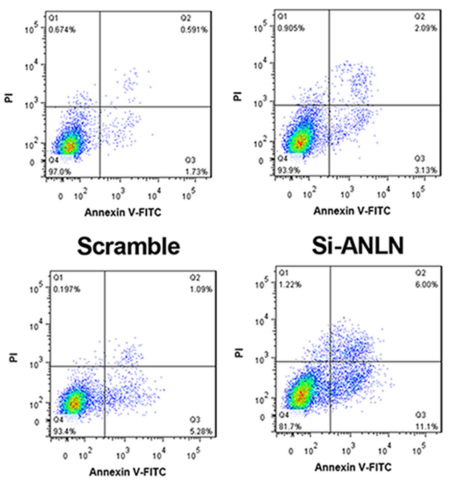

E

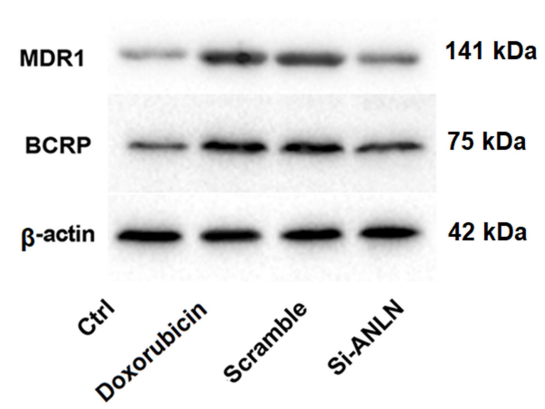

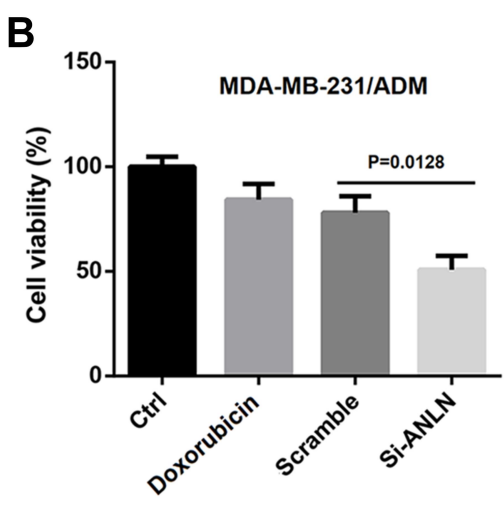
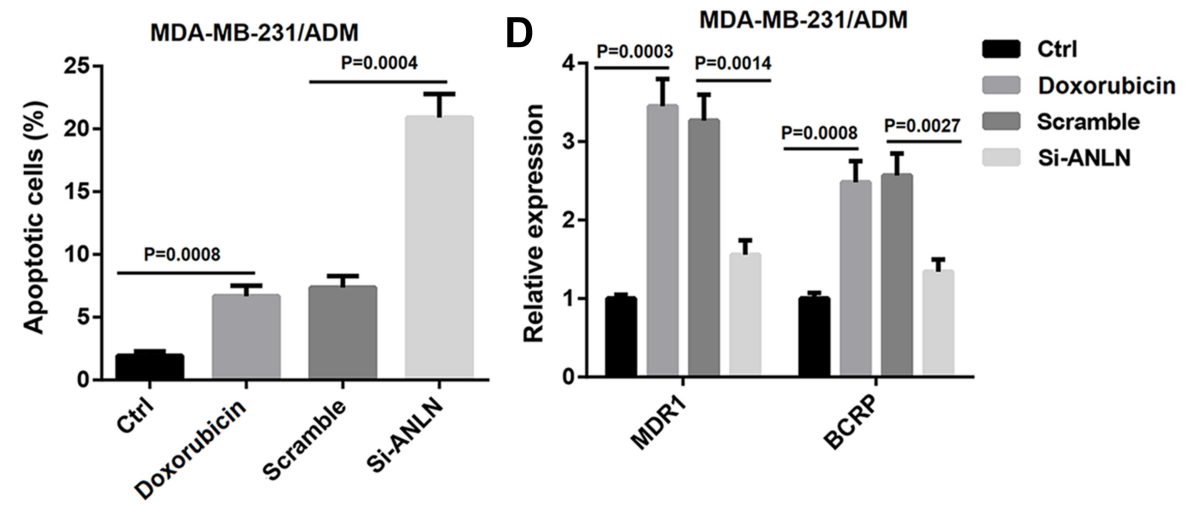

Figure 2 ANLN silencing inhibits doxorubicin resistance in MDA-MB-23I/ADM cells. MDA-MB-23I/ADM cells were transfected with si-ANLN or si-NC. (A) QRT-PCR was performed to estimate ANLN expression in the modified MDA-MB-23I/ADM cells. The wild and modified MDA-MB-23I/ADM cells were treated with doxorubicin. Normal MDA-MB-23I/ADM cells served as control. (B) MTT assay was performed to explore cell viability of the MDA-MB-23I/ADM cells. (C) Flow cytometry was performed to estimate apoptosis of the MDA-MB-23I/ADM cells. (D) The expression of MDRI and BCRP in the MDA-MB-23I/ADM cells was assessed by qRT-PCR. (E) WB was performed to assess the expression of MDRI and BCRP in the MDA-MB-23I/ADM cells.

suppressed by C3 transferase treatment (Figure 4C and D). Taken together, these data validated that ANLN promoted doxorubicin resistance in MDA-MB-231 cells by activating RhoA (Supplementary Figure 1).

\section{Discussion}

Doxorubicin, a well-known anti-cancer drug, is a member of the anthracycline ring antibiotics with broad spectrum of antitumor activity. Doxorubicin diffuses into the cytoplasm of tumor cells and moves to the nucleus in combination with the proteasome. Then, doxorubicin is dissociated and released in the nucleus, which hinders nucleic acid synthesis by acting on
DNA directly. ${ }^{19}$ Doxorubicin interferes with DNA replication, transcription and translation, thereby inhibiting mitosis. It also destroys the spatial structure of DNA and promotes apoptosis of tumor cells by inducing topoisomerase II to cleave DNA molecules. Doxorubicin induces tumor cells to express or release various tumor markers, affects the tumor microenvironment and the immunogenicity of tumor cells, thereby further promoting apoptosis of cancer cells. ${ }^{20-22}$ Doxorubicin is used earlier in breast cancer treatment, and widely used in postoperative adjuvant therapy, neoadjuvant therapy and retreatment after metastasis or recurrence. Doxorubicin alone or combined with other antitumor drugs can effectively control 


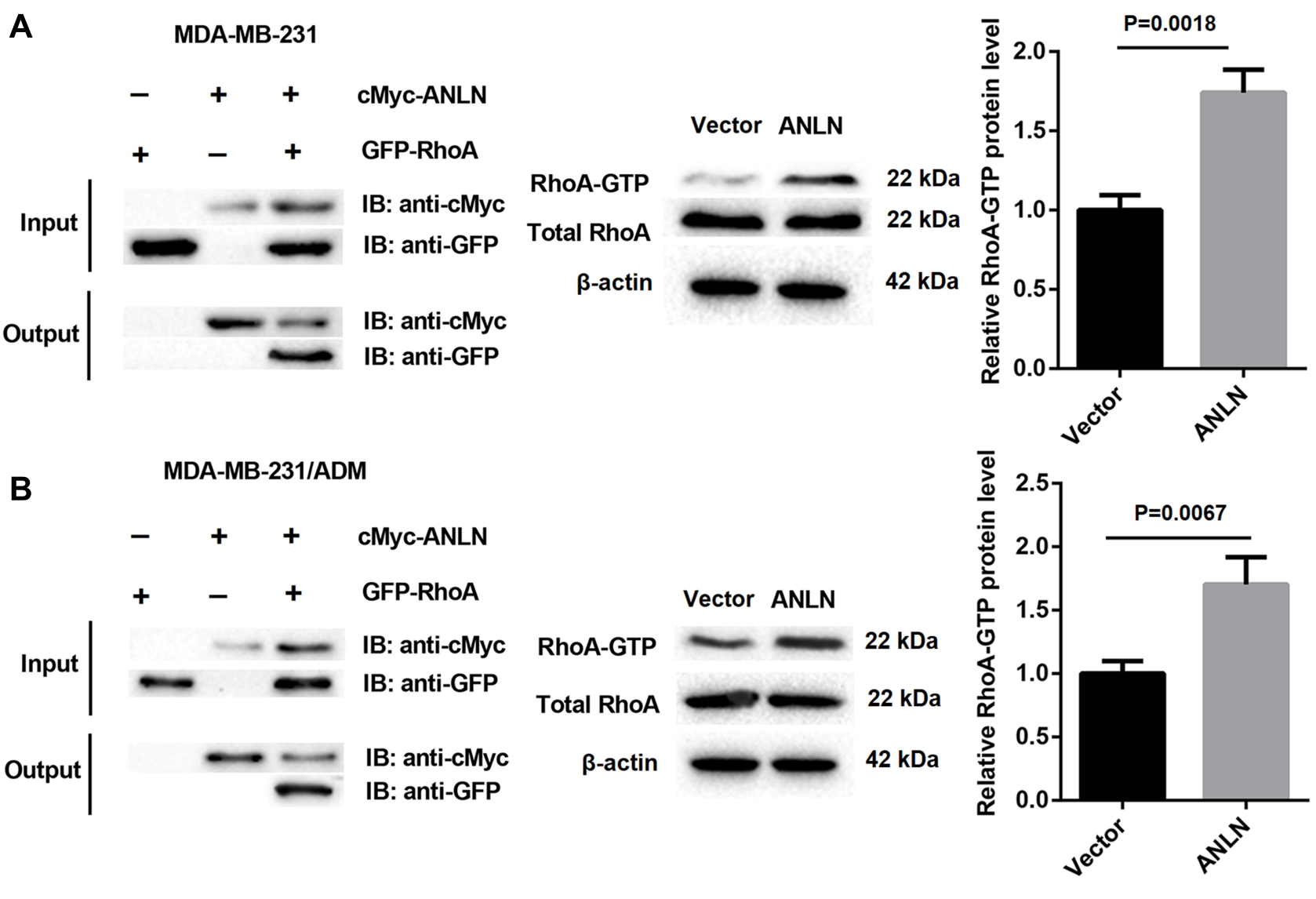

Figure 3 ANLN promotes RhoA activation by interacting with RhoA. (A) Co-IP assay was used to detect the interaction between ANLN and RhoA in MDA-MB-23I cells. (B) Co-IP assay was used to detect the interaction between ANLN and RhoA in MDA-MB-23I/ADM cells.

breast cancer and cervical carcinoma. ${ }^{23,24}$ However, the occurrence of drug resistance is the greatest challenges in breast cancer treatment, and the mechanism is still unclear. Therefore, searching related molecules or genes for drug resistance and revealing the drug resistance mechanism of breast cancer are of great significance for breast cancer treatment.

Previous studies have demonstrated that ANLN is associated with various cancers. Bioinformatics analysis shows that ANLN participates in HBV-related hepatocellular carcinoma. ${ }^{25}$ Surveys such as that conducted by Dai et al show that ANLN down-regulation takes part in the prognostic of breast cancer survival by interacting with $\mathrm{KDR}^{26,27}$ ANLN overexpression leads to poor prognosis and poor clinical outcome to anthracycline-based chemotherapy in breast cancer patients. ${ }^{15}$ In our study, we documented that the doxorubicin resistance in MDA-MB-231/ADM cells was significantly higher than that in MDA-MB-231 cells. ANLN expression was higher in MDA-MB-231/ADM cells than that in MDAMB-231 cells, suggesting that ANLN was associated with doxorubicin resistance in breast cancer cells. The results are in good consistent with the observation that ANLN is highly expressed in breast cancer tissues. ${ }^{14}$ Then, we found that ANLN overexpression led to an increase in cell viability and a decrease in apoptosis of MDA-MB-231 cells. And the gene and protein expression of MDR1 and BCRP was significantly promoted by ANLN up-regulation. MDR1 and BCRP are the drug resistance-related genes. Furthermore, ANLN silencing repressed cell viability and facilitated apoptosis of MDA-MB $-231 / A D M$ cells. And the gene and protein expression of MDR1 and BCRP was obviously decreased in the ANLNsilenced MDA-MB-231/ADM cells. These data demonstrated that ANLN promoted doxorubicin resistance in breast cancer cells.

Many researchers have sought to determine the role of RhoA in the occurrence and development of cancers. Surveys such as that conducted by Kalpana et al show that RhoA knockdown promotes breast cancer metastasis. ${ }^{28} \mathrm{Wnt} 5 \mathrm{a}$ promotes migration and metastasis of breast cancer and esophageal squamous cell carcinoma cells by activating RhoA. ${ }^{29,30}$ Our data verified that ANLN promoted RhoA activation by interacting with RhoA in MDA-MB-231 and MDA-MB-231/ADM cells. ANLN activate Rho signaling 

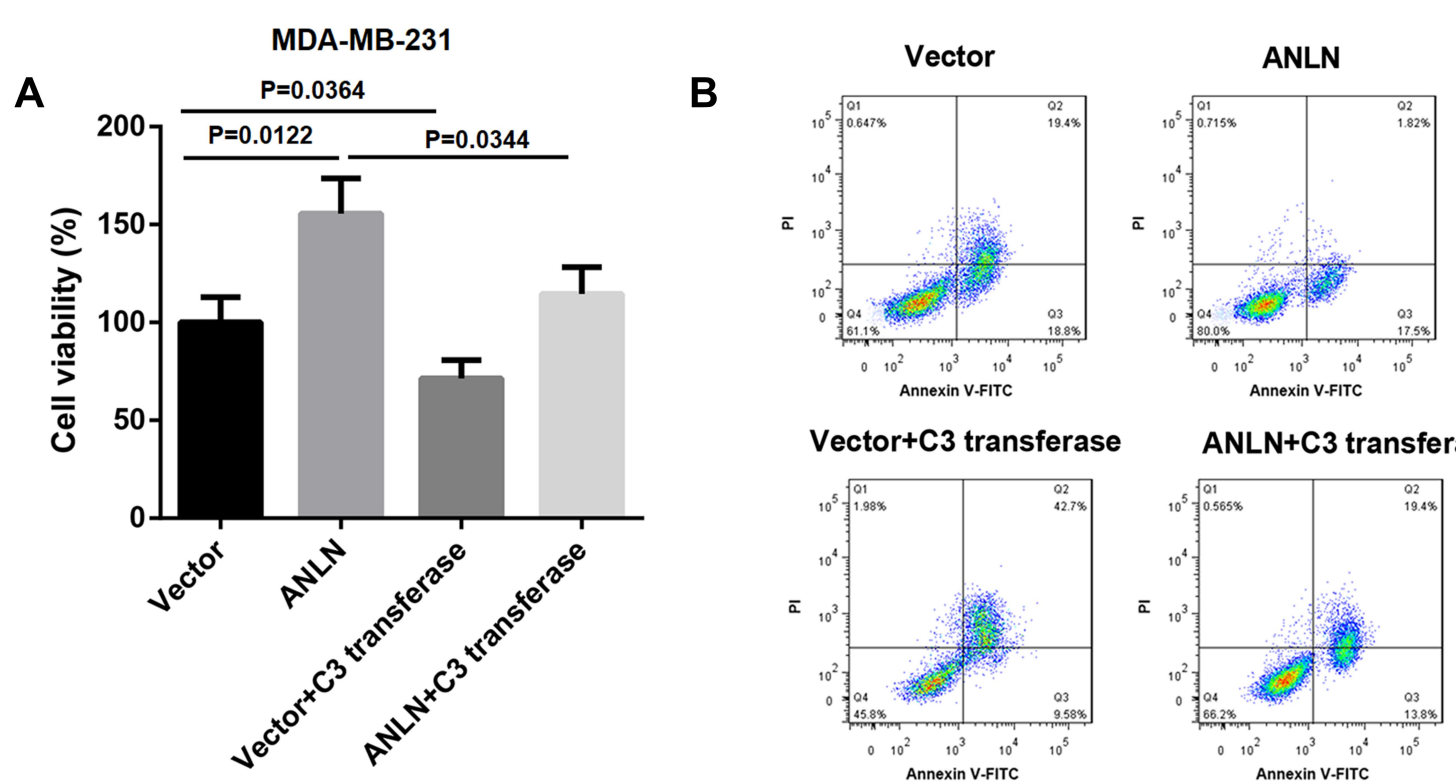

Vector+C3 transferase

\section{ANLN+C3 transferase}
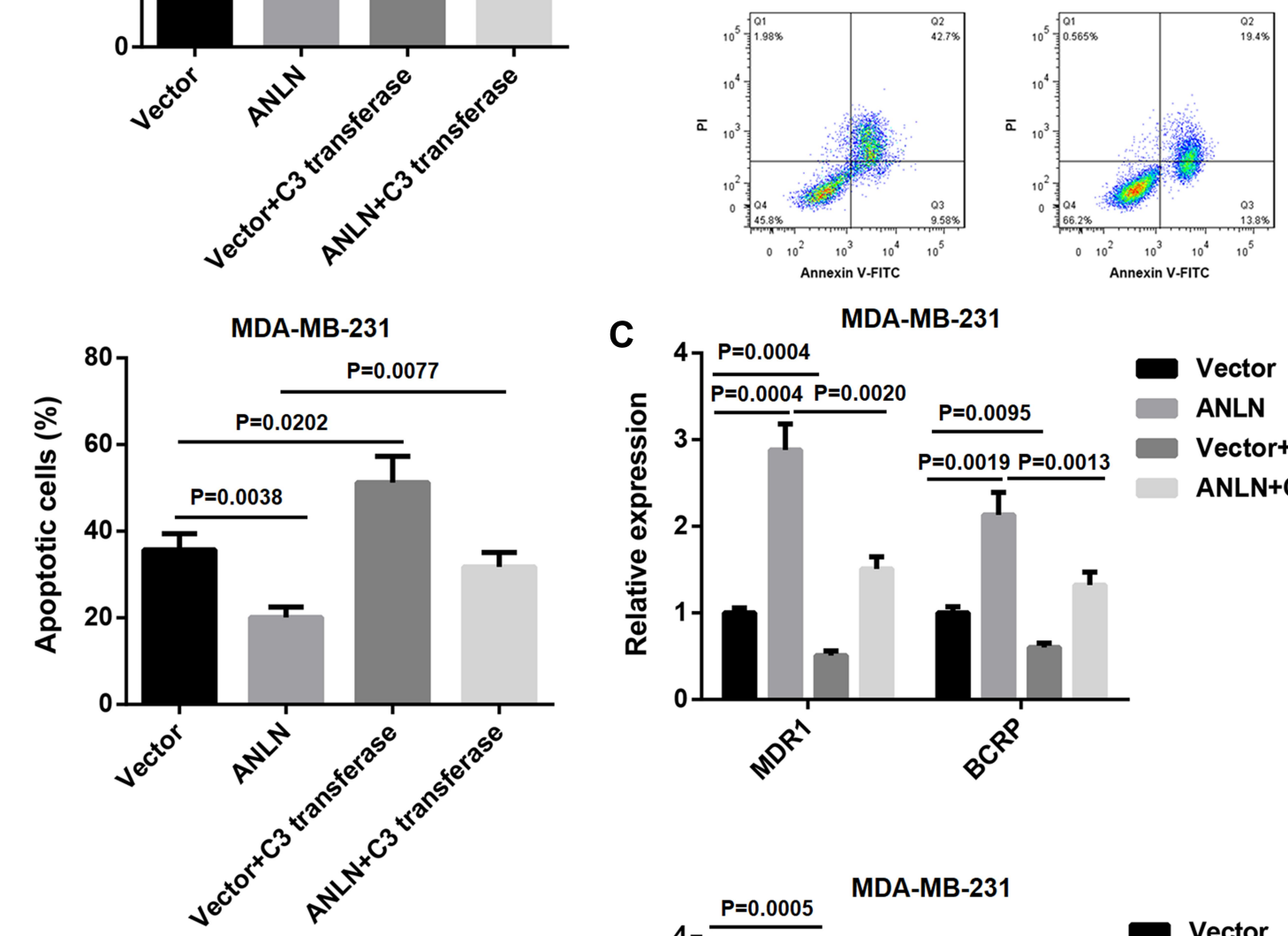

C

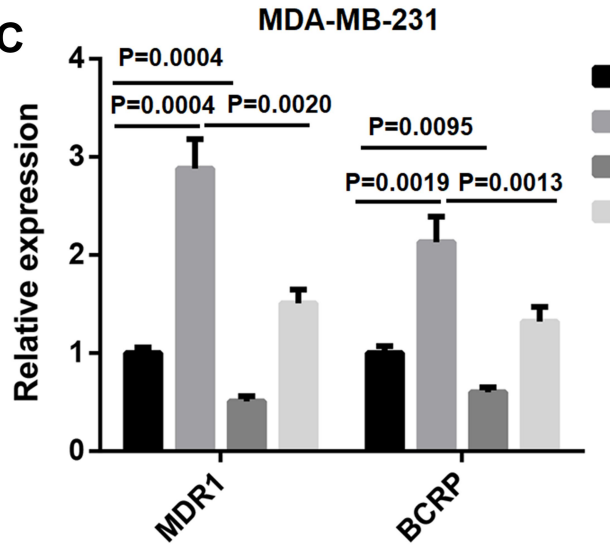

Vector

ANLN

Vector+C3 transferase

ANLN+C3 transferase

D

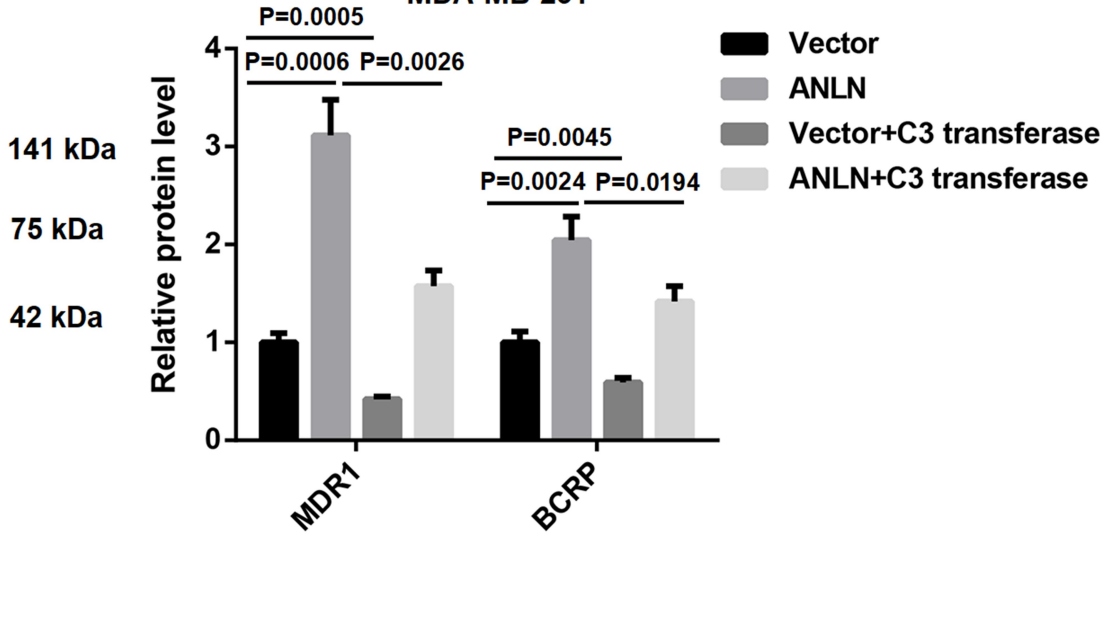

Figure 4 ANLN promotes doxorubicin resistance in MDA-MB-23I cells by activating RhoA. MDA-MB-23I cells were transfected with pcDNA3.I-ANLN or PCDNA3.I-NC. Then, the modified MDA-MB-23I cells were treated with doxorubicin combined with or without C3 transferase. (A) MTT assay was performed to explore cell viability of the MDA-MB-23I cells. (B) Flow cytometry was performed to estimate apoptosis of the MDA-MB-23I cells. (C) The expression of MDRI and BCRP in the MDA-MB-23I cells was assessed by qRT-PCR. (D) WB was performed to assess the expression of MDRI and BCRP in the MDA-MB-23I cells. 
via interaction with RhoA, thereby accelerating reorganization of the actin cytoskeleton. ${ }^{16}$ Whether ANLN can directly bind to other Rho proteins (such as Rac1) is unclear. Moreover, a functional association between ANLN and activation of RhoA remains to be clarified. However, our data suggested that ANLN interacted with RhoA and activated RhoA, thereby promoting doxorubicin resistance in breast cancer cells. To verify this hypothesis, ANLN-upregulated MDA-MB-231 cells were treated with $\mathrm{C} 3$ transferase to inhibit RhoA activity. The results validated that the effect of ANLN overexpression on cell viability, apoptosis and the expression of MDR1 and BCRP were rescued by $\mathrm{C} 3$ transferase treatment. ANLN participates in human lung carcinogenesis by interacting with RhoA. ${ }^{16}$ Inhibition of RhoA in irinotecan-resistant colorectal cancer cells, at least partially, rescues the resistance against irinotecan by inhibiting the expression of MDR $1 .{ }^{11}$ GPR56 promotes drug resistance of colorectal tumor by up-regulating MDR1 expression via a RhoA-mediated mechanism. ${ }^{31}$ MRP1 is multidrug resistance-associated protein, and BCRP is breast cancer resistance protein. Therefore, we presumed that ANLN knockdown may inhibit the expression of MDR1 and BCRP by repressing RhoA. ANLN promoted doxorubicin resistance in breast cancer cells by enhancing the expression of MDR1 and BCRP via a RhoA-mediated mechanism.

From the perspective of molecular, pathological, clinical, therapeutic and prognosis, breast cancer is a highly heterogeneous disease. Traditional clinical staging and patient stratification does not cover all variants of the disease, cannot accurately predict the patient's response to treatment and prognosis. The multi-omics approach accelerates predictive, preventive and personalized medicine practice, which greatly improves the accuracy of predicting responses, stratification of particular patients and eventual personalization of medicine. $^{32,33}$ Moreover, circulating miRNA profiles, as an adjunct to conventional mammography, have great potential in population screening and personalization of breast cancer management. ${ }^{34}$ Fröhlich et al's research has proved that multi-omics data approach can predict premenopausal women as members of high or low breast cancer risk group. ${ }^{35}$ In view of the high incidence and poor prognosis of breast cancer, measures should be taken to prevent the occurrence of breast cancer. For example, the establishment of a population (screening) plan, the establishment of standardized diagnostic methods and targeted preventive measures specifically for pregnancy-associated breast cancer. ${ }^{36}$

\section{Conclusion}

Our studies have shown that the doxorubicin resistance in MDA-MB-231/ADM cells was significantly higher than that in MDA-MB-231 cells. ANLN is highly expressed in MDAMB-231/ADM cells as compared with MDA-MB-231 cells, suggesting that ANLN is closely associated with doxorubicin resistance in breast cancer cells. Furthermore, ANLN overexpression enhances the doxorubicin resistance of MDA-MB -231 cells, whereas ANLN silencing decreases the doxorubicin resistance of MDA-MB-231/ADM cells. In addition, ANLN promotes doxorubicin resistance in breast cancer cells by activating RhoA. Thus, the results imply that ANLN might play a certain role in the doxorubicin resistance of breast cancer and supply a new target for the treatment of breast cancer.

\section{Funding}

This study was supported by the Research Innovation Program for Graduate Students of Central South University (Grant No. 502211901). This study was also supported by the National Key Clinical Specialist Construction Programs of China (Grant No. 2014kll).

\section{Disclosure}

Feng Wang, Zhen Xiang, and Teng Huang are co-first authors for this study. The authors declare no conflicts of interest.

\section{References}

1. Siegel RL, Miller KD, Jemal A. Cancer Statistics, 2017. CA Cancer $J$ Clin. 2017;67(1):7-30. doi:10.3322/caac.21387

2. Abe AR, Enomoto K. Effects of chemotherapy and hormonal therapy for early breast cancer on recurrence and 15-year survival: an overview of the randomised trials. Lancet. 2005;365(9472):1687-1717.

3. Xu H, Zhang XJ, Wang DQ, Xu L, Wang AP. Factors influencing medication-taking behaviour with adjuvant endocrine therapy in women with breast cancer: A qualitative systematic review. $J A d v$ Nurs. 2020;76(2):445-458. doi:10.1111/jan.14253

4. Lambertini M, Boni L, Michelotti A, et al. Ovarian suppression with triptorelin during adjuvant breast cancer chemotherapy and long-term ovarian function, pregnancies, and disease-free survival: a randomized clinical trial. JAMA. 2015;314(24):2632-2640. doi:10.1001/ jama.2015.17291

5. Christensen LA, Finch RA, Booker AJ, Vasquez KM. Targeting oncogenes to improve breast cancer chemotherapy. Cancer Res. 2006;66 (8):4089-4094. doi:10.1158/0008-5472.CAN-05-4288

6. Antoon JW, White MD, Slaughter EM, et al. Targeting NFkB mediated breast cancer chemoresistance through selective inhibition of sphingosine kinase-2. Cancer Biol Ther. 2011;11(7):678-689. doi:10.4161/ cbt.11.7.14903

7. Deng Z, Jia Y, Liu H, et al. RhoA/ROCK pathway: implication in osteoarthritis and therapeutic targets. Am J Transl Res. 2019;11 (9):5324-5331. 
8. Jansen S, Gosens R, Wieland T, Schmidt M. Paving the Rho in cancer metastasis: rho GTPases and beyond. Pharmacol Ther. 2018;183:1-21. doi:10.1016/j.pharmthera.2017.09.002

9. Mokady D, Meiri D. RhoGTPases - A novel link between cytoskeleton organization and cisplatin resistance. Drug Resist Update. 2015;19:22-32. doi:10.1016/j.drup.2015.01.001

10. Yoon C, Cho SJ, Aksoy BA, et al. Chemotherapy resistance in diffuse-type gastric adenocarcinoma is mediated by RhoA activation in cancer stem-like cells. Clin Cancer Res. 2016;22(4):971-983. doi:10.1158/1078-0432.CCR-15-1356

11. Zhang S, Chatterjee T, Godoy C, Wu L, Liu QJ, Carmon KS. GPR56 drives colorectal tumor growth and promotes drug resistance through upregulation of MDR1 expression via a RhoA-mediated mechanism. Mol Cancer Res. 2019;17(11):2196-2207. doi:10.1158/1541-7786. MCR-19-0436

12. Korourian A, Roudi R, Shariftabrizi A, Madjd Z. MicroRNA-31 inhibits RhoA-mediated tumor invasion and chemotherapy resistance in MKN-45 gastric adenocarcinoma cells. Exp Biol Med (Maywood). 2017;242(18):1842-1847. doi:10.1177/1535370217728460

13. Magnusson K, Gremel G, Ryden L, et al. ANLN is a prognostic biomarker independent of $\mathrm{Ki}-67$ and essential for cell cycle progression in primary breast cancer. BMC Cancer. 2016;16(1):904. doi:10.1186/s12885-016-2923-8

14. Zhou W, Wang Z, Shen N, et al. Knockdown of ANLN by lentivirus inhibits cell growth and migration in human breast cancer. Mol Cell Biochem. 2015;398(1-2):11-19. doi:10.1007/s11010-014-2200-6

15. Wang Z, Chen J, Zhong MZ, et al. Overexpression of ANLN contributed to poor prognosis of anthracycline-based chemotherapy in breast cancer patients. Cancer Chemother Pharmacol. 2017;79 (3):535-543. doi:10.1007/s00280-017-3248-2

16. Suzuki C, Daigo Y, Ishikawa N, et al. ANLN plays a critical role in human lung carcinogenesis through the activation of RHOA and by involvement in the phosphoinositide 3-kinase/AKT pathway. Cancer Res. 2005;65(24):11314-11325. doi:10.1158/0008-5472.CAN-05-1507

17. Brinkley BR, Beall PT, Wible LJ. Variations in cell form and cytoskeleton in human breast carcinoma cells in vitro. Cancer Res. 1980;40(9):3118-3129.

18. Hu H, Li S, Cui X, et al. The overexpression of hypomethylated miR-663 induces chemotherapy resistance in human breast cancer cells by targeting heparin sulfate proteoglycan 2 (HSPG2). J Biol Chem. 2013;288(16):10973-10985. doi:10.1074/jbc.M112.434340

19. Moiseeva TN, Bottrill A, Melino G, Barlev NA. DNA damage-induced ubiquitylation of proteasome controls its proteolytic activity. Oncotarget. 2013;4(9):1338-1348. doi:10.18632/oncotarget.1060

20. Zitvogel L, Apetoh L, Ghiringhelli F, Andre F, Tesniere A, Kroemer G. The anticancer immune response: indispensable for therapeutic success? J Clin Invest. 2008;118(6):1991-2001. doi:10.1172/JCI35180

21. Kepp O, Tesniere A, Schlemmer F, et al. Immunogenic cell death modalities and their impact on cancer treatment. Apoptosis. 2009;14 (4):364-375. doi:10.1007/s10495-008-0303-9

22. Beneteau M, Zunino B, Jacquin MA, et al. Combination of glycolysis inhibition with chemotherapy results in an antitumor immune response. Proc Natl Acad Sci USA. 2012;109(49):20071-20076. doi:10.1073/pnas.1206360109

23. Alsaif AA, Hasan TN, Shafi G, et al. Association of multiple drug resistance-1 gene polymorphism with multiple drug resistance in breast cancer patients from an ethnic Saudi Arabian population. Cancer Epidemiol. 2013;37(5):762-766. doi:10.1016/j. canep.2013.04.011

24. Xia Y, Xiao M, Zhao M, et al. Doxorubicin-loaded functionalized selenium nanoparticles for enhanced antitumor efficacy in cervical carcinoma therapy. Mater Sci Eng C Mater Biol Appl. 2020;106:110100. doi:10.1016/j.msec.2019.110100

25. Xie S, Jiang $X$, Zhang J, et al. Identification of significant gene and pathways involved in HBV-related hepatocellular carcinoma by bioinformatics analysis. Peer J. 2019;7:e7408. doi:10.7717/peerj.7408

26. Dai X, Mei Y, Chen X, Cai D. ANLN and KDR are jointly prognostic of breast cancer survival and can be modulated for triple negative breast cancer control. Front Genet. 2019;10:790. doi:10.3389/ fgene. 2019.00790

27. Dai X, Chen X, Hakizimana O, Mei Y. Genetic interactions between ANLN and KDR are prognostic for breast cancer survival. Oncol Rep. 2019.

28. Kalpana G, Figy C, Yeung M, Yeung KC. Reduced RhoA expression enhances breast cancer metastasis with a concomitant increase in CCR5 and CXCR4 chemokines signaling. Sci Rep. 2019;9 (1):16351. doi:10.1038/s41598-019-52746-w

29. Hasan MK, Widhopf GF, Zhang S, et al. Wnt5a induces ROR1 to recruit cortactin to promote breast-cancer migration and metastasis. NPJ Breast Cancer. 2019;5(1):35. doi:10.1038/s41523-019-0131-9

30. Wu X, Yan T, Hao L, Zhu Y. Wnt5a induces ROR1 and ROR2 to activate RhoA in esophageal squamous cell carcinoma cells. Cancer Manag Res. 2019;11:2803-2815. doi:10.2147/CMAR.S190999

31. Ruihua H, Mengyi Z, Chong Z, et al. RhoA regulates resistance to irinotecan by regulating membrane transporter and apoptosis signaling in colorectal cancer. Oncotarget. 2016;7(52):87136-87146. doi:10.18632/oncotarget. 13548

32. Lu M, Zhan X. The crucial role of multiomic approach in cancer research and clinically relevant outcomes. EPMA J. 2018;9 (1):77-102.

33. Janssens JP, Schuster K, Voss A. Preventive, predictive, and personalized medicine for effective and affordable cancer care. EPMA J. 2018;9(2):113-123. doi:10.1007/s13167-018-0130-1

34. Zubor P, Kubatka P, Kajo K, et al. Why the gold standard approach by mammography demands extension by multiomics? Application of liquid biopsy miRNA profiles to breast cancer disease management. Int J Mol Sci. 2019;20(12):12. doi:10.3390/ijms20122878

35. Fröhlich H, Patjoshi S, Yeghiazaryan K, Kehrer C, Kuhn W. Premenopausal breast cancer: potential clinical utility of a multi-omics based machine learning approach for patient stratification. EPMA J. 2018;9(2):175-186. doi:10.1007/s13167018-0131-0

36. Polivka J, Altun I, Golubnitschaja O. Pregnancy-associated breast cancer: the risky status quo and new concepts of predictive medicine. EPMA J. 2018;9(1):1-13. doi:10.1007/s13167-018-0129-7

\section{Publish your work in this journal}

Cancer Management and Research is an international, peer-reviewed open access journal focusing on cancer research and the optimal use of preventative and integrated treatment interventions to achieve improved outcomes, enhanced survival and quality of life for the cancer patient.
The manuscript management system is completely online and includes a very quick and fair peer-review system, which is all easy to use. Visit http://www.dovepress.com/testimonials.php to read real quotes from published authors. 